\title{
Successful Treatment with Rituximab in a Patient with TTP Secondary to Severe ANCA-Associated Vasculitis
}

\author{
Yukari Asamiya ${ }^{1}$, Takahito Moriyama ${ }^{1}$, Mari Takano ${ }^{1}$, Chihiro Iwasaki ${ }^{1}$, Kazuo Kimura $^{1}$, \\ Yukako Ando ${ }^{1}$, Akiko Aoki ${ }^{1}$, Kan Kikuchi ${ }^{2}$, Takashi Takei ${ }^{1}$, \\ Keiko Uchida ${ }^{1}$ and Kosaku Nitta ${ }^{1}$
}

\begin{abstract}
We report a case of thrombotic thrombocytopenic purpura (TTP) secondary to antineutrophil cytoplasmic antibody (ANCA)-associated vasculitis treated by rituximab. TTP secondary to ANCA-associated vasculitis is very rare and has a high mortality rate. We employed rituximab and successfully treated TTP secondary to ANCA-associated vasculitis, because standard therapies, such as steroid therapy, intravenous pulse cyclophosphamide, and repeated plasma exchange (PE), did not suppress her disease activity. This is the first report to suggest that rituximab can achieve complete remission of TTP secondary to ANCA-associated vasculitis.
\end{abstract}

Key words: ANCA-associated vasculitis, TTP, rituximab, renal failure, intrapulmonary hemorrhage, hemodialysis

(Inter Med 49: 1587-1591, 2010)

(DOI: 10.2169/internalmedicine.49.3135)

\section{Introduction}

Antineutrophil cytoplasmic antibody (ANCA)-associated vasculitis is recognized as a multisystem autoimmune disease characterized by ANCA production and small vessel inflammation (1). It's typical clinical manifestations are rapidly progressive glomerulonephritis and the occasional development of intrapulmonary hemorrhage, which is potentially fatal (2).

Thrombotic thrombocytopenic purpura (TTP) is a lifethreatening disease. TTP secondary to ANCA-associated vasculitis is very rare; to the best of our knowledge, only nine cases have been reported. Those nine cases all received intensive therapies such as steroid pulse therapy, intravenous pulse cyclophosphamide (IVCY), and plasma exchange $(\mathrm{PE})$, however, $56 \%$ of them died. Among these previous reports, none of the cases were treated using rituximab (3).

Rituximab is a chimeric monoclonal antibody that targets the B cell-specific CD20 calcium channel, resulting in the depletion of peripheral B cells. Recently, rituximab has been reported in the treatment of TTP resistant to standard thera- pies such as steroids and therapeutic PE (4-6). However, rituximab was employed mainly against idiopathic TTP, and there were few reports discussing treatment of secondary TTP; in particular, TTP with ANCA-associated vasculitis was rare. Here, we report a patient with TTP secondary to myeloperoxidase-antineutrophil cytoplasmic antibody (MPOANCA) associated vasculitis who was treated using rituximab combined with steroid pulse, IVCY and repeated PE. This is the first report of the successful use of rituximab against TTP secondary to ANCA-associated vasculitis.

\section{Case Report}

In 2002, a 59-year-old woman was admitted to our hospital because of marked rapidly progressive renal impairment [creatinine level, $1.56 \mathrm{mg} / \mathrm{dL}$ (normal $0.48-0.79 \mathrm{mg} / \mathrm{dL}$ )], proteinuria and microscopic hematuria, interstitial pneumonia and an elevated MPO-ANCA level of $490 \mathrm{EU}$ (normal < $20 \mathrm{EU})$. Although a renal biopsy was not performed because of hydronephrosis caused by a urinary calculus, she was diagnosed as having MPO-ANCA-associated vasculitis based on the diagnostic criteria of the Ministry of Health, Labor

${ }^{1}$ Department of Medicine, Kidney Center, Tokyo Women's Medical University, Tokyo and ${ }^{2}$ Shimoochiai Clinic, Tokyo Received for publication November 10, 2009; Accepted for publication March 1, 2010 Correspondence to Dr. Takahito Moriyama, takamori@kc.twmu.ac.jp 


\begin{tabular}{|c|c|c|c|c|}
\hline Urinalysis & & Serological test & & \\
\hline Protein & $2+$ & $\lg G$ & $821 \mathrm{~m}$ & $\mathrm{mg} / \mathrm{dL}$ \\
\hline Occult blood & $3+$ & IgM & $128 \mathrm{~m}$ & $\mathrm{mg} / \mathrm{dL}$ \\
\hline Red blood cells & $30-49 / \mathrm{HPF}$ & $\lg A$ & $280 \mathrm{~m}$ & $\mathrm{mg} / \mathrm{dL}$ \\
\hline White blood cells & 5-9/HPF & Serum $\mathrm{CH} 50$ & 48.8 & $\mathrm{U} / \mathrm{mL}$ \\
\hline & & Serum C3 & $104.6 n$ & $\mathrm{mg} / \mathrm{dL}$ \\
\hline Complete blood cell & counts & Serum C4 & $27.1 \mathrm{n}$ & $\mathrm{mg} / \mathrm{dL}$ \\
\hline White blood cells & $11750 / \mu \mathrm{L}$ & Antinuclear antibody & $1: 160$ & \\
\hline Neut & $90.1 \%$ & MPO-ANCA & $119 \mathrm{E}$ & EU \\
\hline Lymph & $6.1 \%$ & PR3-ANCA & $<10 \quad E$ & EU \\
\hline Red blood cells & $297 \times 10^{4} / \mu \mathrm{L}$ & Anti-GBM antibody & $<10 \quad E$ & EU \\
\hline Hemoglobin & $8.9 \mathrm{~g} / \mathrm{dL}$ & & & \\
\hline Platelet & $17.3 \times 10^{4} / \mu \mathrm{L}$ & & & \\
\hline Chemical analysis & & & & \\
\hline Total protein & $5.7 \mathrm{~g} / \mathrm{dL}$ & & & \\
\hline Albumin & $3.1 \mathrm{~g} / \mathrm{dL}$ & & & \\
\hline AST & $27 \mathrm{U} / \mathrm{L}$ & & & \\
\hline LDH & $216 \mathrm{U} / \mathrm{L}$ & & & \\
\hline BUN & $72.6 \mathrm{mg} / \mathrm{dL}$ & & & \\
\hline Creatinine & $3.9 \mathrm{mg} / \mathrm{dL}$ & & & \\
\hline C-reactive protein & $16.4 \mathrm{mg} / \mathrm{dL}$ & & & \\
\hline
\end{tabular}

Figure 1. Laboratory and chest $\mathrm{X}$-ray findings on admission. Chest X-ray image showed a diffuse alveolar pattern. Neut: neutrophilic leukocyte, Lymph: lymphocyte, AST: aspartate aminotransferase, LDH: lactate dehydrogenase, BUN: blood urea nitrogen, MPO-ANCA: myeloperoxidaseantineutrophil cytoplasmic antibody, PR3-ANCA: proteinase 3-antineutrophil cytoplasmic antibody, Anti-GBM antibody: anti-glomerular basement membrane antibody

and Welfare of Japan (7) and treated with high-dose oral prednisolone. Prednisolone was gradually tapered, and she was treated with a maintenance dose of prednisolone of 10 $\mathrm{mg}$ per day and her serum creatinine level was 2.50-2.87 $\mathrm{mg} / \mathrm{dL}$ in 2008.

In February 2009, at the age of 66 years, she was admitted with symptoms of a lack of appetite, fever and respiratory distress. Her laboratory examinations showed the following values: white blood cell count, 1,1750/ $\mathrm{LL}$ (normal 4,000-8,600/ $\mu \mathrm{L}$ ); hemoglobin level, $8.9 \mathrm{~g} / \mathrm{dL}$ (normal 12-16 $\mathrm{g} / \mathrm{dL}$ ); platelet count $17.3 \times 10^{4} / \mu \mathrm{L}$ (normal $15-35 \times 10^{4} / \mu \mathrm{L}$ ), Creactive protein $(\mathrm{CRP}), 16.4 \mathrm{mg} / \mathrm{dL}$ (normal $<0.33 \mathrm{mg} / \mathrm{dL}$ ); MPO-ANCA level, $119 \mathrm{EU}$; creatinine level, $3.89 \mathrm{mg} / \mathrm{dL}$; lactate dehydrogenase (LDH) $216 \mathrm{U} / \mathrm{L}$ (normal 119-229 U/ L); intense proteinuria and microscopic hematuria (Fig. 1). A chest X-ray image showed a diffuse alveolar pattern (Fig. 1). The clinical course of the patient is shown in Fig. 2. On hospital day 2, a bronchoscopy was performed and the patient was diagnosed as having severe pulmonary alveolar hemorrhage. Steroid pulse therapy (methylprednisolone, $500 \mathrm{mg} /$ day $\times 3$ days) was immediately started followed by $40 \mathrm{mg}(1.0 \mathrm{mg} / \mathrm{kg}$ body weight $)$ per day of oral prednisolone based on the guideline of Ministry of Health, Labor and Welfare of Japan (7). On day 7, the chest computed tomography (CT) scan revealed that pulmonary hemorrhage tended to be improved, and her CRP level decreased to $0.4 \mathrm{mg} / \mathrm{dL}$. On the same day fragmented red blood cells (FRC) (schistocytes) were observed in her peripheral blood smear for the first time and she had a high fever. On day 8 , total bilirubin was increased. On day 11 , her platelet count started to decrease to $11.6 \times 10^{4} / \mu \mathrm{L}$ from $15.0-19.0 \times 10^{4} / \mu \mathrm{L}$, LDH increased, her renal dysfunction showed signs of progression (creatinine level, $4.3 \mathrm{mg} / \mathrm{dL}$; blood urea nitrogen level, $114 \mathrm{mg} / \mathrm{dL}$ ) and fluid overload occurred. Then she began receiving intermittent hemodialysis. On day 14, her MPO-ANCA level decreased to 14 EU. The thrombocytopenia was continuously progressive, her platelet count decreased further to $5.0-8.0 \times 10^{4} / \mu \mathrm{L}$. On day 19 , her hemoglobin level decreased to $9.2 \mathrm{~g} / \mathrm{dL}$ from $11.2 \mathrm{~g} / \mathrm{dL}$, a Coombs test was negative, serum haptoglobin level was undetectable. Drug-induced hemolytic anemia and thrombocytopenia were clinically negative and hemodialysis-induced hemolytic anemia and thrombocytopenia were also considered negative because drugs and dialyzer had not been changed during this therapeutic period. Disseminated intravascular coagulation (DIC) was negative based on the diagnostic criteria of the MHLW of Japan (8). On the other hand she had the transient neurological symptoms including headaches, emotional disturbance and personality disorder. A brain CT scan revealed no abnormal findings. The diagnosis of TTP was made based on microangiopathic hemolytic anemia with schistocytes on the peripheral blood smear, high LDH levels, and thrombocytopenia, in addition to clinical features such as fever, organ dysfunctions of renal insufficiency and neurological deficits. Although the patient's condition showed an improvement of ANCA-associated vasculitis, her renal disease was progressive, therefore we regarded that her renal disease was progressed with complicated TTP. PE was started on day 21, which was replaced with 25-30 units of fresh-frozen plasma three times a week, and the ADAMTS 13 activity was decreased at $37.8 \%$ (normal 70-120\%), ADAMTS13 inhibitor level of $<0.5$ Bethesda Units (BU)/ $\mathrm{mL}$ on same day. On day 26, black tarry stools appeared, her blood pressure dropped to 70/42 from 116/66 $\mathrm{mmHg}$ and she required blood transfusion. The upper esophagogastroduodenoscopy and colonoscopy could not be performed immediately, because the patient's general condition was not good. Contrast-enhanced CT scans of her chest, abdomen and pelvis were performed and revealed no acute abnormalities except for diffuse thickening of the gastroduodenal wall. We suspected that disease activity of TTP and ANCAassociated vasculitis was not suppressed by the steroid therapy and PE, thus intravenous pulse cyclophosphamide (IVCY) (cyclophosphamide, $200 \mathrm{mg}$ ) was administered on day 28. The upper esophagogastroduodenoscopy was performed on day 29 and colonoscopy was performed on day 35 , both of them were negative of active bleeding of digestive tract. Eight days after initiation of PE treatment, the ADAMTS13 activity increased to 70.1\% (normal 70-120\%) and ADAMTS 13 inhibitor level was $<0.5 \mathrm{BU} / \mathrm{mL}$. However, her platelet count decreased further and remained at 3.5$5.5 \times 10^{4} / \mu \mathrm{L}$ during the subsequent 10 days. As before, druginduced or hemodialysis treatment-induced thrombocytopenia, or DIC were considered also negative. Consequently, the PE treatment could not be stopped because of 


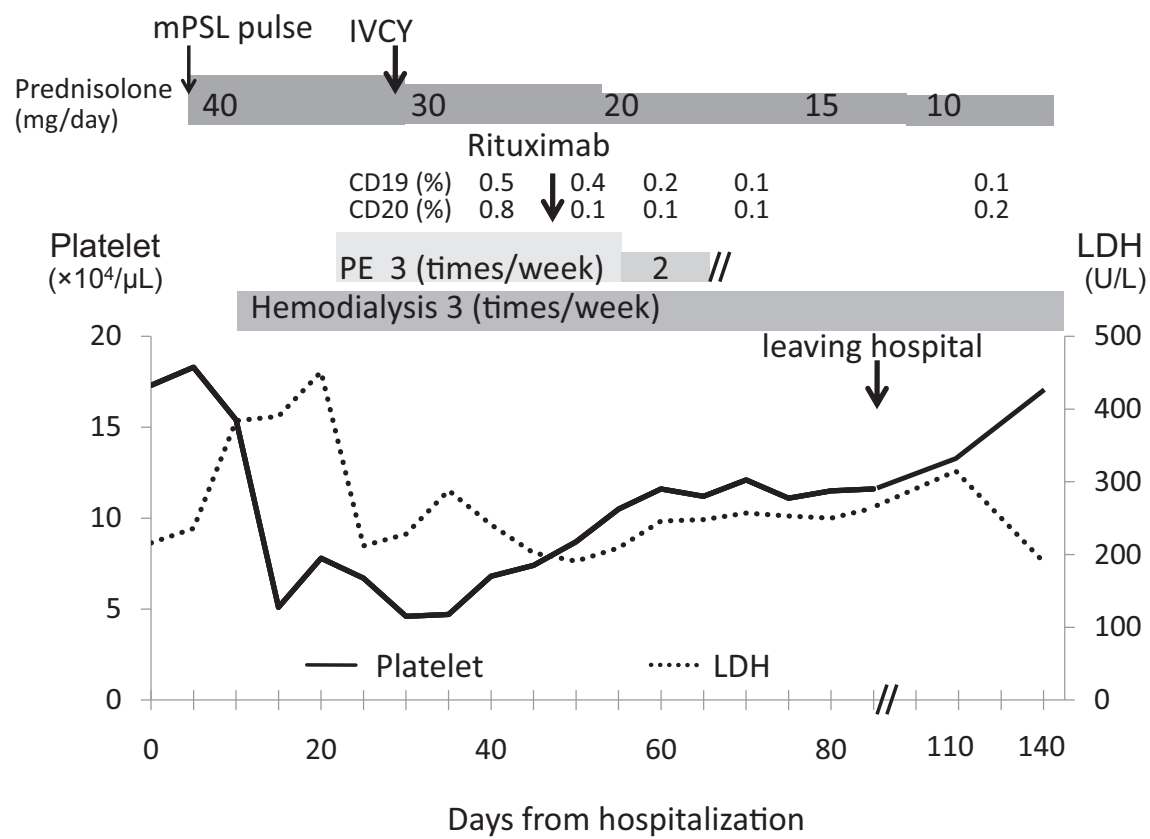

Figure 2. Clinical course of the patient. mPSL: methylprednisolone, IVCY: intravenous cyclophosphamide, LDH: lactate dehydrogenase (normal range 119-229 U/L), PE: plasma exchange

her thrombocytopenia. She was treated with intravenous infusions of rituximab $\left(200 \mathrm{mg}, 150 \mathrm{mg} / \mathrm{m}^{2}\right)$ on day 51 . Thereafter, her platelet count increased and exceeded $10.0 \times$ $10^{4} / \mu \mathrm{L}$, the schistocytes disappeared in her peripheral blood, and the hemolytic parameters were nearly or completely normalized. One week after initiation of rituximab administration, PE was tapered to twice a week and 12 days after initiation of rituximab administration, PE was stopped. Although she remained dependent on dialysis, her critical care status improved and she achieved a complete clinical remission. Her platelet count increased to $17.5 \times 10^{4} / \mu \mathrm{L}$ at 90 days after rituximab treatment.

\section{Discussion}

TTP is divided into congenital, idiopathic, and secondary presenting in a variety of triggering clinical situations including autoimmune disorders, post-hematopoietic stem cell transplantation, malignancy, pregnancy, drug ingestion, and infection. TTP secondary to ANCA-associated vasculitis is a very rare disease. To the best of our knowledge, only nine cases have been previously reported; of these cases, only three had MPO-ANCA-associated vasculitis. Although these previous cases received intensive therapies, such as highdose corticosteroids, IVCY and repeated PE, 5 of the 9 patients $(56 \%)$ with ANCA-associated vasculitis died; moreover, 2 of the 3 patients $(67 \%)$ with MPO-ANCA associated vasculitis died. In all of these cases, the TTP was thought to have developed during the active course of vasculitis (3). Similar to these previous reports, TTP developed in the present case. At the time of this admission in 2009 her Birmingham Vasculitis Activity Score (BVAS 2003) (9) was as high as 39 points, in comparison to 21 points in 2002 .
Regarding the pathogenesis of TTP, platelet activation, thrombosis and thrombocytopenia are associated with unusually large von-Willebrand factor (vWF) multimers. In most patients with idiopathic TTP, a deficiency or dysfunction of ADAMTS13, which is an enzyme that cleaves vWF multimers to smaller forms, is observed (10-12), and IgG-class antibody to ADAMTS13, which acts as an inhibitor, is detected, and unusually large vWF multimers are typically present. PE acts to replace ADAMTS13 and removes circulating ADAMTS13 inhibitors (13), and has improved the survival rate of these patients from less than $10 \%$ to more than $80 \%(14,15)$.

In contrast, in patients with secondary TTP, the ADAMTS 13 activity varied from a marked reduction to normal levels, while the ADAMTS13 inhibitor was not detected in the sera of half of the patients (16). Therefore, most of these patients do not respond to PE (17) and the mortality rate is relatively high, compared with that among patients with idiopathic TTP. In the present case, ADAMTS13 activity was slightly decreased to $37.8 \%$ and the level of ADAMTS13 inhibitor was normal; thus, PE alone was suspected to be insufficient. Kameda et al reported that secondary TTP can be caused by the formation of excessive numbers of unusually large $\mathrm{vWF}$ multimers in endothelial cells stimulated by cytokines such as tumor necrosis factor-alpha, interleukin (IL)-8, and IL6 (17). Indeed, the vascular endothelial damage that occurs in TTP secondary to ANCA-associated vasculitis is observed in the capillaries and small arteries (18). Nagai et al reported that repeated PE might be needed in addition to immunosuppressive therapy until the vascular endothelium can be repaired (3). We treated the present patient who had TTP secondary to ANCA-associated vasculitis using steroid pulse, IVCY, and repeated PE; however, her disease activity 
was not suppressed and her renal disease was progressive with complicated TTP.

Rituximab is currently used in patients with TTP who have an unsatisfactory response to standard therapies such as steroids and therapeutic PE (4-6). In addition, some studies have reported that rituximab is an effective remission induction therapy for refractory ANCA-associated vasculitis $(19,20)$. Therefore, the present patient was treated with rituximab. Rituximab is a chimeric monoclonal antibody against CD20 that selectively depletes B-cells in the circulation and lymphoid tissues. B cells play several important roles including autoantibody production, cytokine secretion, antigen presentation, and a co-stimulatory effect (21). The effect of rituximab against idiopathic TTP is thought to be associated with the elimination of an important source of CD20-positive B cells that produce ADAMTS13 inhibitors (22). Moreover, the efficacy of rituximab has also been reported in patients with secondary TTP associated with collagen disease and normal ADAMTS13 inhibitor levels. Rituximab is thought to reduce excessive cytokine production through B cell depletion (17). These reports allowed us to suspect that the repair of endothelial damage enabled by the rituximab-induced suppression of cytokine secretion allowed the number of unusually large vWF multimers to be reduced, thereby suppressing the disease activity in the present case.

No evidence of the optimal dosage for rituximab treat- ment for TTP was available in any previous report. The standard dose of rituximab for the treatment of non-Hodgkin lymphoma is $375 \mathrm{mg} / \mathrm{m}^{2}$ administered weekly. However, some data suggests that a lower dose $(100 \mathrm{mg}$ intravenous infusion weekly for 4 weeks) may be sufficient for the treatment of TTP because the CD20-positive B cell burden in patients with TTP is much lower than in patients with nonHodgkin lymphoma (23). We used the intravenous infusion of a single dose of rituximab, which resulted in the sufficient depletion of the CD20 and CD19 levels in the present patient. An additional infusion of rituximab was not required. This dosage was beneficial for stopping PE and achieving remission. Moreover, no adverse effects of rituximab, such as fever, chills, nausea, or the rarer adverse effects of urticaria, orthostatic hypotension, or bronchospasm (24), were observed in the present case.

In summary, we encountered a patient with TTP secondary to MPO-ANCA-associated vasculitis, which is a very rare disease. This is the first report to suggest that a single dose of rituximab can enable a complete remission of this disease without any adverse effects. However, no concrete evidence of the optimal timing or dose of rituximab required for the treatment of this disease is available, and a large prospective study is needed to confirm the usefulness of rituximab against TTP secondary to MPO-ANCA-associated vasculitis.

\section{References}

1. Jones RB, Ferraro AJ, Chaudhry AN, et al. A multicenter survey of rituximab therapy for refractory antineutrophil cytoplasmic antibody-associated vasculitis. Arthritis Rheum 60: 2156-2168, 2009.

2. Oh JS, Lee CK, Kim YG, et al. Clinical features and outcomes of microscopic polyangiitis in Korea. J Korean Med Sci 24: 269-274, 2009.

3. Nagai K, Kotani T, Takeuchi $T$, et al. Successful treatment of thrombotic thrombocytopenic purpura with repeated plasma exchange in a patient with microscopic polyangiitis. Mod Rheumatol 18: 643-646, 2008.

4. Ling HT, Field JJ, Blinder MA. Sustained response with rituximab in patients with thrombotic thrombocytopenic purpura: a report of 13 cases and review of the literature. Am J Hematol 84: 418-421, 2009.

5. Scaramucci L, Niscola P, Palumbo R, et al. Rapid response and sustained remission by rituximab in four cases of plasmaexchange-failed acute thrombotic thrombocytopenic purpura. Int J Hematol 89: 389-399, 2009.

6. Bresin E, Gastoldi S, Daina E, et al. Rituximab as pre-emptive treatment in patients with thrombotic thrombocytopenic purpura and evidence of anti-ADAMTS13 autoantibodies. Thromb Haemost 101: 233-238, 2009.

7. Sakai H, Kurokawa K, Koyama A, et al; Committee for the Guidelines on Diagnosis and Therapy of Rapidly Progressive Glomerulonephritis. The guidelines for the management of rapidly progressive glomerulonephritis. Jpn J Nephrol 44: 55-82, 2002 (in Japanese).

8. Wada H, Gabazza EC, Asakura H, et al. Comparison of diagnostic criteria for disseminated intravascular coagulation (DIC): diagnostic criteria of the International Society of Thrombosis and Hemo- stasis and of the Japanese Ministry of Health and Welfare for overt DIC. Am J Hematol 74: 17-22, 2003.

9. Yumura W, Ito C. Activity of ANCA related vasculitis and the value of organ-injury. J Jpn Coll Angiol 49: 63-73, 2009.

10. Fujimura $\mathrm{Y}$, Matsumoto $\mathrm{M}$, Yagi $\mathrm{H}$, et al. Von Willebrand factorcleaving protease and Upshaw-Schulman syndrome. Int J Hematol 75: 25-34, 2002.

11. Tsai HM, Lian EC. Antibodies to von Willebrand factor-cleaving protease in acute thrombotic thrombocytopenic purpura. N Engl J Med 339: 1585-1594, 1998.

12. Furlan M, Robles R, Solenthaler M, et al. Deficient activity of von Willebrand factor-cleaving protease in chronic relapsing thrombotic thrombocytopenic purpura. Blood 89: 3097-3103, 1997.

13. Allford SL, Hunt BJ, Rose P, Machin SJ. Guidelines on the diagnosis and management of the thrombotic microangiopathic haemolytic anaemias. Br J Haematol 120: 556-573, 2003.

14. Sarode R, Gottschall JL, Aster RH, McFarland JG. Thrombotic thrombocytopenic purpura: early and late responders. Am J Hematol 54: 102-107, 1997.

15. Rock GA, Shumak KH, Buskard NA, et al. Comparison of plasma exchange with plasma infusion in the treatment of thrombotic thrombocytopenic purpura. Canadian Apheresis Study Group. N Engl J Med 325: 393-397, 1991.

16. Fujimura Y. Progress on TTP/HUS diagnosis with analysis of VWF-cleaving protease activation. Nippon Naika Gakkai Zasshi 93: 451-459, 2004 (in Japanese).

17. Kameda T, Dobashi H, Kittaka K, et al. Two cases of refractory thrombotic thrombocytopenic purpura associated with collagen vascular disease were significantly improved by rituximab treatment. Clin Rheumatol 26: 2159-2162, 2007. 
18. Moake JL. von Willebrand factor, ADAMTS-13, and thrombotic thrombocytopenic purpura. Semin Hematol 41: 4-14, 2004.

19. Jones RB, Ferraro AJ, Chaudhry AN, et al. A multicenter survey of rituximab therapy for refractory antineutrophil cytoplasmic antibody-associated vasculitis. Arthritis Rheum 60: 2156-2168, 2009.

20. Stasi R, Stipa E, Del Poeta G, et al. Long-term observation of patients with anti-neutrophil cytoplasmic antibody-associated vasculitis treated with rituximab. Rheumatology (Oxford) 45: 1432-1436, 2006.

21. Hasegawa M, Hasegawa M. B lymphocyte. Nihon Rinsho Meneki Gakkai Kaishi 28: 300-308, 2005 (in Japanese).
22. Willis MS, Bandarenko N. Relapse of thrombotic thrombocytopenic purpura: is it a continuum of disease? Semin Thromb Hemost 31: 700-708, 2005.

23. Zaja F, Battista ML, Pirrotta MT, et al. Lower dose rituximab is active in adults patients with idiopathic thrombocytopenic purpura. Haematologica 93: 930-933, 2008.

24. Winkler U, Jensen M, Manzke O, et al. Cytokine-release syndrome in patients with B-cell chronic lymphocytic leukemia and high lymphocyte counts after therapy with an anti-CD20 monoclonal antibody (Rituximab, IDEC-C2B8). Blood 94: 2217-2224, 1999.

(C) 2010 The Japanese Society of Internal Medicine http://www.naika.or.jp/imindex.html 